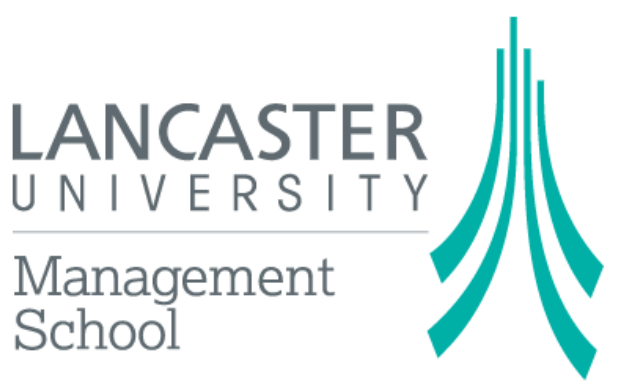

Economics Working Paper Series

2014/005

\title{
Strategic Choice of Sharing Rules in Collective Contests
}

Pau Balart, Sabine Flamand, Orestis Troumpounis

The Department of Economics

Lancaster University Management School

Lancaster LA1 4YX

UK

(C) Authors

All rights reserved. Short sections of text, not to exceed two paragraphs, may be quoted without explicit permission, provided that full acknowledgement is given. 


\title{
Strategic Choice of Sharing Rules in Collective Contests
}

\author{
Pau Balart* Sabine Flamand ${ }^{\dagger} \quad$ Orestis Troumpounis $^{\ddagger}$
}

August 29, 2014

\begin{abstract}
Competition between groups often involves prizes that have both a public and a private component. The exact nature of the prize not only affects the strategic choice of the sharing rules determining its allocation but also gives rise to an interesting phenomenon not observed when the prize is either purely public or purely private. Indeed, we show that in the two-groups contest, for most degrees of privateness of the prize, the large group uses its sharing rule as a mean to exclude the small group from the competition, a situation called monopolization. Conversely, there is a degree of relative privateness above which the small group, besides being active, even outperforms the large group in terms of winning probabilities, giving rise to the celebrated group size paradox.
\end{abstract}

Keywords: collective rent seeking, strategic choice of sharing rules, group size paradox, monopolization

*Department of Economics, Universidad Carlos III de Madrid, Calle Madrid 126, 28903 Getafe, Spain, e-mail: pbalart@eco.uc3m.es

${ }^{\dagger}$ Nova School of Business and Economics, Campus de Campolide, P-1099-032, Lisbon, Portugal. Phone: +351213801655 , e-mail: sabine.flamand@novasbe.pt

${ }_{\ddagger}^{\ddagger}$ Department of Economics, Lancaster University, Bailrigg, Lancaster, LA1 4YX, United Kingdom, e-mail: o.troumpounis@lancaster.ac.uk 


\section{Introduction}

Collective contests where group members organize and compete for a prize are a common phenomenon: research and development races, procurement contests, funds to be allocated among different departments within a university, regions within a country or countries in the EU, projects allocated among different divisions of a firm, party members who participate in pre-electoral campaigns, or even sport and art contests. Often, one can interpret the prizes sought by competing groups as a mixture between a public and a private good. Regional governments competing for a budget typically use the latter to provide both monetary transfers and local public goods. Likewise, prizes in research and development races involve both reputational and monetary benefits for the winning team. More generally, any contest between groups for a monetary reward can be considered as involving a public good component as long as the winners enjoy some benefits in terms of status, reputation, or satisfaction related to the victory.

An important feature of collective contests is that groups' performance depends on individual contributions of their members. University departments usually receive their funds depending on the publication record of the department, which is determined by the individual publications of its members. Therefore, groups face the need of coordinating and establishing some rules regarding their internal organization. As pointed out by previous literature, one of the most relevant decisions in this respect concerns the way the private component of the prize is shared among groups members in case of victory. ${ }^{1}$

In this paper we consider a standard model of collective rent seeking allowing for the contested prize to be a mixed public-private good. We analyze how the nature of the prize affects the strategic choice of sharing rules and thus individual incentives to contribute to their group effort, which may ultimately give rise to the occurrence of two phenomena: i) monopolization and ii) the group size paradox (GSP henceforth). In the context of

\footnotetext{
${ }^{1}$ Starting with Nitzan (1991), the literature has considered both exogenous and endogenous sharing rules, while it has assumed that the choice of such rules may occur under either public or private information. For a recent survey on prize-sharing rules in collective rent seeking, see Flamand and Troumpounis (2014).
} 
two-groups contests, monopolization refers to a situation in which one group retires from the competition (Ueda, 2002). While such a situation can never be an equilibrium when the contestants are individuals, it may occur in collective rent seeking to the extent that the members of the active group are still competing for the private part of the prize within the group. On the contrary, the GSP refers to a situation in which a smaller group outperforms a larger one in terms of winning probabilities. The notion of the GSP dates back to the seminal work by Olson (1965), who stressed the severity of the free-rider problem within large groups. In the context of collective rent seeking with endogenous sharing rules, the occurrence of the GSP has been studied by Nitzan and Ueda (2011).

We contribute to the literature on the strategic choice of sharing rules by analyzing the case of intermediate degrees of privateness of the contested prize. This literature has focused extensively on the extreme case of a purely private contested prize, while considering that the choice of sharing rules may be either restricted or unrestricted. If it is restricted, a situation to which we refer as "bounded" meritocracy, the private component of the prize can be allocated among group members at most proportionally to individual contributions (Baik, 1994; Lee, 1995; Noh, 1999; Ueda, 2002). On the contrary, if the choice of sharing rules is unrestricted (i.e., meritocracy is "unbounded"), the group may decide to allocate the private part of the prize more than proportionally to the individual efforts of its members, in which case worse-performing group members pay a transfer to better-performing group members (Baik and Shogren, 1995; Baik and Lee, 1997, 2001; Lee and Hyeong Kang, 1998; Gürtler, 2005). ${ }^{2}$ As we discuss later, it turns out that if the groups were able to choose whether to restrict the level of meritocracy of their sharing rules, they would (weakly in some instances) never do so regardless of the exact nature of the prize. Hence, the very fact that the unconstrained choice of sharing rules in both groups always arises endogenously as an equilibrium constitutes a justification for departing from the assumption of bounded meritocracy. The other extreme case where

\footnotetext{
${ }^{2}$ Recent literature has considered cost-sharing in collective contests for purely public prizes, which can also be interpreted as within-group transfer schemes (Nitzan and Ueda, 2014; Vazquez, 2014). Nitzan and Ueda (2014) provide several examples of situations involving transfers among members of a group, in contexts such as labor unions, ethnic conflicts, or academic institutions.
} 
the prize is a pure public good — and thus sharing rules do not apply — has been analyzed by Baik $(1993,2008)$. However, as we pointed out with the above examples, prizes in collective contests are likely to involve both a public and private component.

We show that allowing for a mixed public-private prize in collective rent seeking dramatically alters the nature of competition and incentives with respect to the extreme cases of a purely private or public contested prize. In particular, we show that unbounded meritocracy yields monopolization for most intermediate degrees of privateness of the prize. In contrast, monopolization never arises in collective contests when the prize is purely private or purely public, even when allowing for meritocracy to be unbounded (Baik and Lee, 1997).

Our analysis also contributes to the understanding of the occurrence of the GSP, which has been studied recently in the context of collective contests with a mixed publicprivate prize by Esteban and Ray (2001) and Nitzan and Ueda (2011). While Esteban and Ray (2001) assume exogenous and fully egalitarian group sharing rules, Nitzan and Ueda (2011) extend their analysis by considering the possibility of endogenous sharing rules. However, they assume that the information regarding such rules is private, hence their choice does not involve a strategic interaction between groups. In this paper, we consider an additional factor that may further affect the occurrence of the GSP, namely the strategic choice of sharing rules in a setup of perfect information. In the imperfect information setup of Nitzan and Ueda (2011), a higher level of meritocracy in a group induces higher levels of individual effort by its members. In our perfect information setup, a more meritocratic sharing rule not only induces higher levels of individual effort by that group's members, but it also discourages effort by the other group's members. While the GSP always arises in the case of exogenous and egalitarian sharing rules analyzed by Esteban and Ray (2001) (except for a purely public good), it never does so in the context of endogenous sharing rules under imperfect information considered by Nitzan and Ueda (2011). By taking into account the additional strategic effects induced by the assumption of perfect information combined with the one of unbounded meritocracy, we show that 
the GSP arises whenever the prize is sufficiently private. ${ }^{3}$

While it is often reasonable to think of groups' internal organization (sharing rules) as private information, our analysis applies to many other cases that are better described by a situation of perfect information where the decisions regarding sharing rules are potentially strategic. ${ }^{4}$ Think for instance of university departments competing for funds, with the departments allocating part of these funds among their members according to a publicly known incentive scheme. More generally, any competition taking place among groups belonging to a common organization is often likely to involve common knowledge of the individual incentive schemes within each group. Likewise, it is not unreasonable to consider that in some instances, firms have some knowledge regarding the incentives schemes of their competitors.

Two distinct effects relating to group size have to be taken into account in order to understand our results. For a given level of privateness, the most direct effect of group size is that it yields better odds at winning the competition between groups, since there is a larger number of potential contributors. Put otherwise, if all individuals exert the same level of effort, then the aggregate contribution is greater for the large group, and so is its probability of winning the competition between groups. This is what we call the aggregate effort effect, and it favors the large group regardless of the nature of the contested prize. However, there is an additional effect related to group size that penalizes the large group: a more numerous group implies that the private part of the prize has to be divided among more individuals. That is, if the good is (at least partially) private,

\footnotetext{
${ }^{3}$ While the two previous works provide further results on the influence of a convex cost of effort on the elimination of the GSP, the introduction of a strategic choice of sharing rules obliges us to restrict ourselves to the linear cost case. A convex cost of effort penalizes higher levels of individual effort, hence it works against the occurrence of the GSP. Corchón (2007) provides an intuition for the latter: "Intuitively, it is clear that Olson's conjecture cannot hold if costs rise very quickly with effort: for instance if costs are zero up to a point, say $\bar{G}$ where they jump to infinity, all agents will make effort $\bar{G}$ and smaller groups will exert less effort than large ones." Our conjecture is then that monopolization should also hold with convex costs, while the GSP should be less likely.

${ }^{4}$ In fact, in the literature on collective rent seeking and sharing rules the assumption of perfect information as in this paper is the usual one. To the best of our knowledge, the only papers analyzing the case of private information are Baik and Lee (2007, 2012), Nitzan and Ueda (2011), Baik (2014) and Nitzan and Ueda (2014). Monopolization never arises in that context, even when considering intermediate degrees of privateness of the contested prize.
} 
a larger group size also implies a lower individual share of the prize in case of victory. This is what Nitzan (1991) calls the group-size deterrence effect. Clearly, this effect does not apply to the public component of the prize given the non-exclusion and non-rivalry properties of public goods. ${ }^{5}$

The relative size of these two effects ultimately determines the emergence of monopolization and the GSP. As the group-size deterrence effect is stronger the higher the degree of privateness of the prize, while the aggregate effort effect is independent of the nature of the prize, it follows that the aggregate effort effect dominates the size deterrence effect whenever the public component of the prize is large enough. Indeed, if the prize is sufficiently public (but not a pure public good), and if the groups can decide upon the meritocracy level of their sharing rules without any constraint, then the large group takes advantage of its size by selecting a very meritocratic sharing rule. This increases the individual contributions by its members while discouraging individual contributions by the small group's members, to the point of excluding the small group from the competition - that is, monopolization occurs. However, when the degree of privateness of the prize increases, the size deterence effect gets larger, acting as a counterbalancing power to the aggregate effort effect. When the prize is sufficiently private, the small group has an interest in actively taking part in the competition. As the degree of privateness increases further, the size deterrence effect exacerbates, thereby decreasing the attractiveness of a potential victory from the perspective of the large group's members. As a result, the large group implements a less meritocratic rule than the small group, inducing relatively lower levels of effort by its members, which ultimately drives the emergence of the GSP.

We start the analysis with the strategic choice of group sharing rules under unbounded meritocracy, that yields the main results of the paper. If the prize is sufficiently public (but not purely public), monopolization occurs and the small group retires from the contest. If the prize is sufficiently private the large group cannot exploit its size advantage,

\footnotetext{
${ }^{5}$ Relaxing the property of non-rivalry by assuming that the public part of the prize is congestionable does not qualitatively change our results (see footnotes 11 and 12).
} 
so that the GSP occurs. We then proceed with the analysis of the strategic choice of group sharing rules under bounded meritocracy. We show that when the allocation of the prize among group members can be at most proportional to individuals' relative contributions, then neither the large nor the small group can implement a sharing rule that allows them to fully exploit their respective size advantage (i.e., size deterrence for the small group and aggregate effort for the large group). As a consequence, neither monopolization nor the GSP arises when meritocracy is bounded. In other words, the large (small) group needs to select more than proportional levels of meritocracy in order to fully take advantage of the aggregate effort (size deterrence) effect. Yet, one can show that the members of both groups are generally strictly better off when the choice of their respective sharing rules is restricted and the strength of competition reduced accordingly. Therefore, even though the restricted choice of sharing rules in both groups would never arise endogenously as an equilibrium, it turns out that in most instances, it Pareto-dominates the case of unbounded meritocracy.

\section{The Model}

There are two groups $i=A, B$ of size $n_{i} \in \mathbb{N}_{+}$and without loss of generality $A$ is the large group (i.e., $n_{A}>n_{B}$ ). All individuals within a group are identical and the cost of exerting effort is linear. ${ }^{6}$ The valuation of the contested (divisible) good is the same for all individuals, and is denoted by $V$. Individuals' choice of effort within each group takes place simultaneously given the sharing rules. Individuals are risk neutral, and the expected utility of individual $k=1, \ldots, n_{i}$ in group $i$ is given by

\footnotetext{
${ }^{6}$ We abstract from the possibility of intra-group heterogeneity regarding lobbying effectivity, which can be reflected in different valuations of the prize by the members. One can ask whether a group whose members have highly unequal interests in the collective action will be more or less active. The literature has provided contrasted answers to the latter question, due to the difference in the assumed form of the effort cost function (see the discussion in Nitzan and Ueda (2013) and the references they cite). Assuming very weak and plausible restrictions on the form of the effort cost functions, Nitzan and Ueda (2013) show that if a group competes for a prize with sufficiently many rivals or with a very superior rival, unequal stakes among the members can enhance its performance.
} 


$$
E U_{k i}=\left\{\left[\alpha_{i} \frac{e_{k i}}{E_{i}}+\left(1-\alpha_{i}\right) \frac{1}{n_{i}}\right] p+(1-p)\right\} \frac{E_{i}}{E_{A}+E_{B}} V-e_{k i}
$$

where $E_{i}=\sum_{j=1}^{n_{i}} e_{j i}$ denotes the aggregate effort of group $i$ and $p \in[0,1]$ measures the degree of privateness of the contested prize $(p=0$ corresponds to the case of a pure public good while $p=1$ corresponds to the case of a pure private good). The sharing rule $\alpha_{i}$ is the relative weight given to meritocracy as opposed to egalitarianism in the division of the private part of the prize within the corresponding group. As long as $\alpha_{i}>0$, high performers in group $i$ receive a larger share of the prize than low performers, while if $\alpha_{i}=0$, the private part of the prize is shared equally among group members regardless of their relative contributions.

Previous literature on the strategic choice of sharing rules has considered both the cases of $\alpha_{i} \in[0,1]$ (Baik, 1994; Lee, 1995; Noh, 1999; Ueda, 2002) and $\alpha_{i} \in[0, \infty)$ (Baik and Shogren, 1995; Baik and Lee, 1997, 2001; Lee and Hyeong Kang, 1998). The restriction that $\alpha_{i} \in[0,1]$ implies that the allocation of the private part of the prize among group members can be at most proportional to individual contributions. On the contrary, a value of $\alpha_{i}$ larger than one implies that the private part of the prize is allocated more than proportionally to relative effort within group $i$. More precisely, when $\alpha_{i}>1$, group $i$ collects $-\left(1-\alpha_{i}\right) \frac{p}{n_{i}} \frac{E_{i}}{\left(E_{A}+E_{B}\right)} V$ from each of its members and then allocates $\alpha_{i} \frac{p}{n_{i}} \frac{E_{i}}{\left(E_{A}+E_{B}\right)} V$ according to relative effort within the group. Consequently, for values of $\alpha_{i}$ larger than one, an individual exerting no effort pays a transfer to the group members who exert positive effort. Thus, if the efforts of the active group members are sufficiently high, expected utility may be negative for an individual exerting no effort. ${ }^{7}$ In this paper we analyze both the restricted and the unrestricted choice of sharing rules.

Our game consists in the strategic choice of the groups' sharing rules, followed by the simultaneous choice of individuals' effort levels. The equilibrium concept is subgame perfection in pure strategies. In the effort stage we focus on within-team symmetric

\footnotetext{
${ }^{7}$ Cost-sharing in collective contests for purely public prizes can also be interpreted in terms of withingroup transfers (Nitzan and Ueda, 2014; Vazquez, 2014).
} 
equilibria.

\section{Effort Stage}

Before analyzing the strategic choice of group sharing rules, we first solve the last stage of the game. At the effort stage, groups' members decide their level of individual effort by maximizing (1) subject to the condition of non-negative efforts. The equilibrium is characterized in Proposition 1, which essentially extends the results of Ueda (2002) and Davis and Reilly (1999) by allowing for a mixed public-private prize.

Proposition 1. Let $\chi_{i}\left(\alpha_{A}, \alpha_{B}\right)=\left(1-\alpha_{i}\right) p\left(n_{i} n_{j}-n_{j}\right)-\left(1-\alpha_{j}\right) p\left(n_{i} n_{j}-n_{i}\right)-n_{i} n_{j}(1-p)-$ $p n_{i}$ with $i=A, B$ and $j \neq i$. There exists a unique within-team symmetric equilibrium in pure strategies in the effort subgame characterized as follows:

- When $\chi_{i}\left(\alpha_{A}, \alpha_{B}\right)<0$ for all $i=A, B$ :

$$
\hat{e_{i}}=\frac{\Lambda_{i} \Phi_{i}}{n_{i}\left[n_{j} p+n_{i}\left(2 n_{j}(1-p)+p\right)\right]^{2}} V
$$

- When $\chi_{i}\left(\alpha_{A}, \alpha_{B}\right) \geq 0$ for group $i=A, B$ :

$$
\tilde{e}_{i}=0
$$

with $\Lambda_{i}=\left[n_{j}(1-p)+p\right]\left[n_{i}(1-p)+p+\left(n_{i}-1\right) p \alpha_{i}\right]+\left(n_{j}-1\right)\left[n_{i}(1-p)+p\right] p \alpha_{j}$ and $\Phi_{i}=n_{j} p\left(1-\alpha_{i}\right)+n_{i}\left[n_{j}\left(1-p\left(1-\alpha_{i}+\alpha_{j}\right)\right)+p \alpha_{j}\right]$.

Expression $\chi_{i}\left(\alpha_{A}, \alpha_{B}\right)$ determines the occurrence of monopolization. More specifically, when $\chi_{i}\left(\alpha_{A}, \alpha_{B}\right) \geq 0$, group $i$ retires from the contest. This condition coincides with the monopolization condition for a purely private prize found by Ueda (2002). As can be seen from the analytical expression of $\chi_{i}\left(\alpha_{A}, \alpha_{B}\right)$, the more meritocratic is the sharing rule of a group, the more likely that its members are active, as it provides higher incentives to exert effort. Conversely, more egalitarian sharing rules exacerbate free-riding incentives, so that individual effort eventually drops to zero (i.e., monopolization arises). Furthermore, the 
more meritocratic the sharing rule of its opponent, the more likely that a group retires from the competition.

When only one group is active, its members compete in a standard Tullock contest with equilibrium effort increasing in the relative meritocracy of the sharing rule and decreasing in the size of the group. When both groups are active (i.e., $\chi_{i}\left(\alpha_{A}, \alpha_{B}\right)<0$ for all $i=A, B)$, equilibrium effort in each group is increasing in the relative meritocracy of its sharing rule and in the value of the prize. In such case, the GSP may occur according to the following definition:

Definition 1. The GSP arises if and only if $E_{A}<E_{B}$.

Given the contest success function used for the competition between groups, the previous definition is equivalent to the small group facing a higher probability of winning than the large group. Using the above equilibrium for exogenous sharing rules, we obtain our first result regarding the GSP:

Proposition 2. With exogenous sharing rules,

- For $p=0$, the GSP never arises.

- For $p \in(0,1]$, the GSP arises if and only if

$$
\alpha_{A}<\frac{n_{A}-n_{B}+2 \alpha_{B} n_{A}\left(n_{B}-1\right)}{2 n_{B}\left(n_{A}-1\right)}
$$

Esteban and Ray (2001) assume that the private part of the prize is shared on an egalitarian basis among group members regardless of their individual contribution, and find that with a linear cost of effort, the GSP arises for all values of $p$ except for the extreme case of $p=0$ (i.e., when the prize is purely public). ${ }^{8}$ Introducing the possibility of (exogenous) group-specific sharing rules leads to a more nuanced result. In particular, as

\footnotetext{
${ }^{8}$ Pecorino and Temimi (2008) modify the model of Esteban and Ray (2001) to a standard public good setting, and show that their results are robust to the presence of small fixed costs of participation in the case of a pure public good, but not in the case of a fully rival good. Furthermore, when the degree of rivalry is sufficiently high, the introduction of small fixed costs of participation implies that collective action must break down in large groups.
} 
can be seen from (2), for any degree of privateness of the good, the less (more) meritocratic the sharing rule of the large (small) group, the more likely that the GSP arises.

\section{Unbounded Meritocracy}

We now consider the first stage of the game where the two groups $A$ and $B$ choose their sharing rules $\left(\alpha_{A}, \alpha_{B}\right)$, and we allow for unbounded levels of meritocracy (i.e., $\alpha_{i} \in[0, \infty)$ for $\left.i=A, B\right)$. As individuals are identical within groups, we assume that in each group, the choice regarding the sharing rule is made by the representative individual by maximizing his expected utility function. That is, the problem of the representative member $k$ in group $i$ consists in choosing the sharing rules $\alpha_{i}$ that maximizes (1) subject to the equilibrium efforts found in Proposition 1.

As the sharing rules are irrelevant when the prize is a pure public good, we assume in what follows that $p>0$. While the equilibrium concept is subgame perfection, in our formal results we present the equilibrium choice of sharing rules, as effort levels chosen in stage two can be derived directly from Proposition 1.

Proposition 3. Let $p_{1}=\frac{n_{B}\left(n_{A}-n_{B}-1\right)}{1+n_{B}\left(n_{A}-n_{B}-1\right)}$. If the choice of sharing rules is unrestricted:

- If $0<p \leq p_{1}$, only group $A$ is active and the continuum of sharing rules in the subgame perfect equilibrium is given by

$$
\begin{aligned}
-\alpha_{A} & =\frac{n_{A}}{n_{A}-1}\left[\frac{1-p}{p}+\frac{\left(n_{B}-1\right) \alpha_{B}+1}{n_{B}}\right] \\
-\alpha_{B} & \in\left[\frac{n_{B}(1-p)+p}{p}, \frac{n_{B}\left[n_{A}(1-p)+3 p-2\right]-2 p}{\left(n_{B}-1\right) p}\right]
\end{aligned}
$$

- If $p>p_{1}$, both groups are active and the sharing rule for $i=A, B$ in the unique subgame perfect equilibrium is given by

$$
\alpha_{i}=\frac{n_{i}}{\left(n_{i}-1\right)} \frac{2 n_{i} n_{j}\left(n_{i}-1\right)+A_{i} p+B_{i} p^{2}}{p\left[2 n_{i} n_{j}-p\left(n_{i}\left(2 n_{j}-1\right)-n_{j}\right)\right]}
$$

where $A_{i}=n_{i} n_{j}\left(9-4 n_{i}\right)-n_{j}\left(n_{j}+2\right)-2 n_{i}$ and $B_{i}=n_{i} n_{j}\left(2 n_{i}-7\right)+n_{j}\left(n_{j}+3\right)+3 n_{i}-2$. 
The above equilibrium sharing rules for the case of a purely private prize $(p=1)$ coincide with the ones found by Baik and Lee (1997). Allowing for the contested prize to have both a public and a private component may lead to the inactivity of the small group, as stated in the following corollary:

Corollary 1. If the choice of sharing rules is unrestricted monopolization arises for all $p \in\left(0, p_{1}\right]$.

Under unbounded meritocracy, monopolization arises for any strictly positive degree of privateness smaller than the threshold $p_{1}{ }^{9}$ This is in stark contrast with both the cases of a purely public and purely private prize, for which monopolization never occurs. However, the reasons for which both groups are active in each of these two cases differ sharply.

One can identify two opposite effects of group size driving the previous results. On the one hand, there is an aggregate effort effect. As groups' performance is additive in the contributions of their members, a larger group size implies better odds at winning the competition between groups. In other words, conditional on having identical levels of individual effort across groups, the large group has a higher probability of winning the prize. On the other hand, there is a group-size deterrence effect (Nitzan, 1991). Contrary to its public component, the private part of the prize is subject to exclusion and rivalry, and these properties are increasingly harmful the greater the size of the group. Clearly, the size deterrence effect vanishes when the good is purely public, while it exacerbates when the degree of privateness increases.

For degrees of privateness lower than $p_{1}$ the size deterrence effect is too weak to compensate for the aggregate effort effect. Given that the level of meritocracy is unbounded, the large group is able to select a sharing rule that places great emphasis on relative effort (i.e., $\alpha_{A}>1$ for all $p<p_{1}$ ), inducing high levels of effort by its members. In turn, the combination of high individual effort levels and a large number of contributors in

\footnotetext{
${ }^{9}$ The results for $p=0$ are not analyzed here. They can be obtained from Baik (2008).
} 
the large group discourages the small group's members from actively taking part in the competition (all $\alpha_{B}$ in the equilibrium interval guarantees the small group's inactivity).

When the degree of privateness exceeds $p_{1}$, the size deterrence effect is strong enough to compensate for the aggregate effort effect, so that it is optimal for the small group to select a highly meritocratic sharing rule (i.e., $\alpha_{B}>1$ for all $p>p_{1}$ ) in order to induce positive levels of effort by its members. Therefore, for degrees of privateness larger than $p_{1}$, both groups are active. In other words, even when the prize is purely private, the size deterrence effect never dominates the aggregate effort effect to the point of excluding the large group from the competition. That is, monopolization is always such that the small group is inactive and occurs if and only if the prize is sufficiently public.

The absence of a size deterrence effect when the prize is purely public suggests that we should also observe monopolization in that case. However, as shown by Baik (2008), when the prize is purely public both groups are active and face the same probability of winning the prize. ${ }^{10}$ Our results thus highlight an interesting discontinuity regarding the occurrence of monopolization in collective rent seeking. Although the presence of a mixed public-private prize constitutes an intermediate possibility between the extreme cases of a purely private or public prize, it does not yield intermediate results in terms of group performance. When the level of meritocracy is unbounded, an arbitrarily small and negligible degree of privateness of the prize is sufficient to allow the large group to address free-riding among its members and exclude the small group from the competition. If the prize is purely public, however, this coordination mechanism is not available to the large group, which is then unable to exploit its size advantage. That is, even though there is no deterrence effect when the prize is a pure public good, the large group cannot exploit the aggregate effort effect due to the large free-riding incentives among its members. Conversely, if the prize exhibits an arbitrarily small degree of privateness, the large group uses its sharing rule as a coordination device to address free-riding among its members.

\footnotetext{
${ }^{10}$ On pure public goods, see also Riaz et al. (1995), Ursprung (1990) and Katz et al. (1990), among others. They have shown that with a pure public good, a group with larger membership attains a winning probability larger than or equal to that of a smaller group. Neither monopolization nor the GSP arise in such cases.
} 
In particular, by selecting a highly meritocratic sharing rule, the members of the large group are able to coordinate at the minimum level of individual contributions such that the small group retires from the contest.

Observe, furthermore, that such preemptive behaviour by the large group occurs for most degrees of privateness, as the threshold $p_{1}$ is in fact very close to one. ${ }^{11}$ Again, such a coordination mechanism among the members of the large group is possible to the extent that the sharing rule $\alpha_{i}$ may exceed one, in which case an inactive group member has to pay a transfer to the other (active) group members. As we shall see in the next section, the unavailability of this mechanism prevents the occurrence of monopolization whenever the choice of sharing rules is subject to the restriction that $\alpha_{i} \in[0,1]$.

We saw that when $p \geq p_{1}$, the size deterrence effect is large enough as compared to the aggregate effort effect so that it is optimal for the small group's members to actively take part in the competition. As the degree of privateness increases further, so does the strength of the size deterrence effect, ultimately giving rise to the GSP. In particular, when the degree of privateness exceeds the threshold $p_{G S P}$, the small group, besides being active, outperforms the large group in terms of winning probabilities.

Proposition 4. With an unrestricted choice of sharing rules, the GSP arises if and only if $p>p_{G S P}=\frac{2 n_{A} n_{B}}{1+2 n_{A} n_{B}} .12$

As explained above, size deterrence is a consequence of the rivalry and exclusion properties of private goods. Therefore, increasing the degree of privateness exacerbates

\footnotetext{
${ }^{11}$ Notice that the numerical value of $p_{1}$ is large and very close to one as the size of the groups increases, and/or when there is a large difference between group sizes. For instance, if $n_{A}=15$ and $n_{B}=7$ then $p_{1}=0.98$. However, this critical value of the degree of privateness can be rescaled by assuming that the public good is congestible. This could be captured by a parameter $c \in[0,1)$ such that the utility attributed to the public good by any member of group $i$ is $(1-p) V / n_{i}^{c}$. In that case the critical value $p_{1}$ is given by $p_{1}^{c}=\frac{n_{B}\left[n_{A}^{c}\left(1+n_{B}\right)-n_{A} n_{B}^{c}\right]}{n_{A}^{c} n_{B}\left(1+n_{B}\right)-n_{B}^{c}\left(n_{A}^{c}+n_{A} n_{B}\right)}$, which for the previous example rescales $p_{1}$ to $p_{1}^{c}=0.76$ for $c=0.6$. In order to avoid unnecessary complications in the main text, we consider the case of $c=0$.

${ }^{12}$ The numerical value of $p_{G S P}$ is large and very close to one as the size of the groups increases, and/or when there is a large difference between group sizes. For instance, if $n_{A}=15$ and $n_{B}=7$ then $p_{G S P}=0.99$. Again, this value can be rescaled if we assume that the public good is congestible. In that case $p_{G S P}^{c}=\frac{n_{A} n_{B}\left[n_{B}^{c}+2 n_{A} n_{B}^{c}-n_{A}^{c}\left(1+2 n_{B}\right)\right]}{n_{B}^{c}\left[n_{A}^{c}\left(n_{A}-n_{B}\right)+n_{A}\left(1+2 n_{A}\right) n_{B}\right]-n_{A}^{1+c} n_{B}\left(1+2 n_{B}\right)}$, which for the previous example yields $p_{G S P}^{c}=0.95$ for $c=0.6$.
} 
the size deterrence effect. This drives the emergence of the GSP for levels of privateness above $p_{G S P}$.

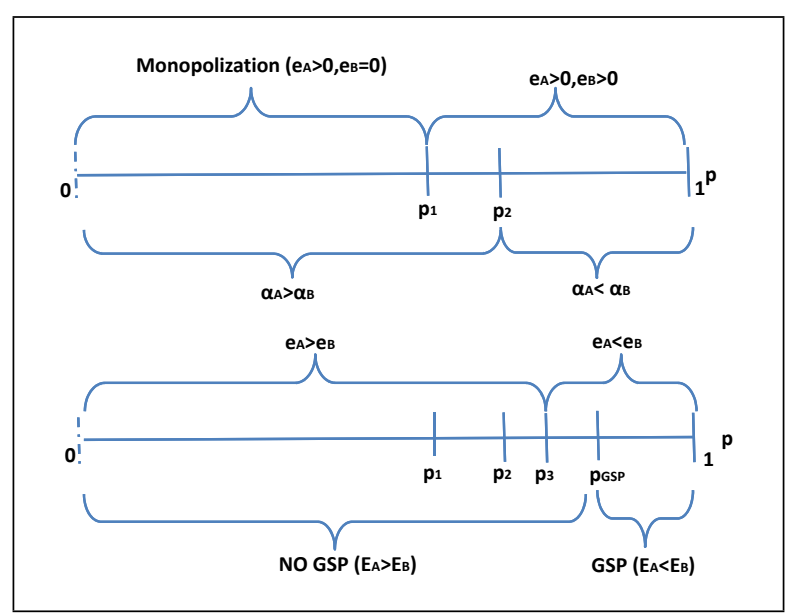

Figure 1: Simultaneous choice of sharing rules

Figure 1 is useful to illustrate the emergence of the GSP in further detail. When the degree of privateness exceeds $p_{1}$, both groups are active. Furthermore, the large group implements a more meritocratic rule than the small one $\left(\alpha_{A}>\alpha_{B}\right)$ as long as $p \leq p_{2} \cdot{ }^{13}$ As a consequence of the size deterrence effect, the relative meritocracy of the large group's sharing rule is strictly decreasing in the degree of privateness of the prize. As the good gets relatively more private $\left(p>p_{2}\right)$, it is the small group that implements the most meritocratic sharing rule $\left(\alpha_{A}<\alpha_{B}\right)$. However, returns to effort are still higher for the large group's members, so that they exert higher individual effort until a yet larger degree of privateness is reached. From $p_{3}$ on, the relative level of meritocracy in both groups is such that the small group's members exert higher individual effort than the members of the large group, i.e., from $p>p_{3}$ it holds that $e_{B}>e_{A}$. Finally, when the degree of privateness reaches $p_{G S P}$, the larger amount of individual effort exerted by the small group's members is sufficient to compensate for the smaller number of contributors, so that the GSP arises.

\footnotetext{
${ }^{13}$ To avoid introducing more notation, we do not define formally the thresholds $p_{2}$ and $p_{3}$ presented in the interpretation of our results. They can be easily calculated and one can show that $p_{1}<p_{2}<p_{3}<$ $p_{G S P}$.
} 


\section{Bounded Meritocracy}

We now solve the model assuming that the level of meritocracy is bounded. That is, the sharing rules must be such that the allocation of the private part of the prize among group members is at most proportional to relative contributions, i.e., $\alpha_{i} \in[0,1]$ for $i=A, B$. As we will see, neither monopolization nor the GSP arise in that case.

Proposition 5. If the choice of sharing rules is restricted:

- If $0<p \leq \stackrel{\circ}{p}$, both groups are active and the sharing rules in the unique subgame perfect equilibrium are given by $\alpha_{A}=\alpha_{B}=1$

- If $p>\stackrel{\circ}{p}$, both groups are active and the sharing rules in the unique subgame perfect equilibrium are given by:

$$
\begin{aligned}
& -\alpha_{A}=\frac{n_{A}^{2} n_{B}\left[2 n_{B}(1-p)+p\right](1-p)-n_{B} p\left[2 n_{B}(1-p)+p\right]-n_{A}\left[2 n_{B}(1-2 p) p+p^{2}+n_{B}^{2}(2+p(5 p-7))\right]}{2\left(n_{A}-1\right) n_{B}\left[n_{B}(1-p)+p\right] p} \\
& -\alpha_{B}=1
\end{aligned}
$$

The above equilibrium sharing rules for the case of a purely private prize $(p=1)$ coincide with the ones found by Lee (1995). ${ }^{14}$ The constraint affecting the sharing rules is always binding for the small group, while it is binding for the large group at low levels of privateness. ${ }^{15}$ When $p>\stackrel{\circ}{p}$ the strength of the size deterrence effect induces the large group to select a sharing rule such that individual rewards within the group are less than proportional to relative contributions. As the constraints are binding in equilibrium, restricting the choice of sharing rules clearly alter our previous results.

Proposition 6. If the choice of sharing rules is restricted, neither monopolization nor the GSP occurs for any $p \in[0,1]$.

With bounded levels of meritocracy neither the large nor the small group can implement a sharing rule that enables them to fully exploit their size advantage (i.e., size

\footnotetext{
${ }^{14}$ The equilibrium (restricted) sharing rules for the case of $n$ groups are provided by Ueda (2002).

${ }^{15}$ The analytical expression of the threshold $\stackrel{\circ}{p}$ can be found in the appendix.
} 
deterrence for the small group and aggregate effort for the large group). As a consequence, neither monopolization nor the GSP occurs. In other words, the large (small) group needs to select more than proportional levels of meritocracy in order to fully take advantage of the aggregate effort (size deterrence) effect. In a nutshell, it is the combination of unbounded levels of meritocracy and intermediate degrees of privateness of the contested prize that drives the occurrence of the two phenomena studied in this paper: monopolization and the GSP.

The restriction on the levels of meritocracy sets an upper bound on the strength of competition by preventing the possibility of transfers within the groups. If the prize is sufficiently private so that both groups are active (i.e., $p>p_{1}$ ), it can be shown that both groups' members are strictly better off when the choice of sharing rules is restricted. Although the GSP never takes place - and thus the large group is more likely to win the competition - the small group's members are better off in the restricted version of the game given their reduced effort levels. If the prize is sufficiently public (i.e., $p<p_{1}$ ), we saw that under unbounded meritocracy the large group selects highly meritocratic sharing rules so as to exclude the small group from the competition. In this case, we showed that there exists a continuum of equilibria where the large group selects the minimum value of $\alpha_{A}\left(\alpha_{B}\right)$ guaranteeing the inactivity of the small group for any admissible value of $\alpha_{B}$ (Proposition 3). The small group's members are clearly better off under bounded meritocracy as they actively take part in the competition and thus achieve strictly positive payoffs. Whether the large groups' members prefer the restricted version of the game depends on the exact equilibrium realization. Despite being inactive, the small group's members may select a high level of meritocracy, thereby obliging the large groups' members to increase their own level of meritocracy and thus their effort levels. In such case, the large group's members prefer the choice of sharing rules to be restricted. Conversely, if the small group's members select a low level of meritocracy out of the equilibrium support, the large group's members are better off under unbounded meritocracy. 
As the decision whether to restrict the level of meritocracy is ultimately a group's internal issue, it is natural to endogenize it. To do so, one could add a third stage to our game where prior to the choice of their sharing rules, the two groups would simultaneously decide whether to play the restricted or unrestricted version of the game. It can then be shown that both groups selecting unbounded levels of meritocracy is always a subgame perfect equilibrium for all values of privateness. Moreover, the version of the game where both groups choose to restrict their choice of sharing rules never arises as an equilibrium given their incentives to deviate and lift the restriction. ${ }^{16}$ Hence, we believe that our analysis of the strategic choice of sharing rules under unbounded meritocracy and the related results on the occurrence of monopolization and the GSP are of interest.

\section{Discussion}

We have shown that the strategic choice of sharing rules has important implications regarding the occurrence of both monopolization and the GSP. In particular, the feasible meritocracy levels of the sharing rules and the degree of privateness of the contested prize are key. On the one hand, both monopolization and the GSP can only arise with unbounded levels of meritocracy, while on the other hand, the degree of privateness of the prize affects these two phenomena in an opposite way. In particular, while monopolization occurs for low degrees of privateness, the GSP arises for high degrees of privateness. In turn, this is a consequence of the two different size effects that shape the equilibrium of the game: the aggregate effort effect (which benefits the large group) and the size deterrence effect (which benefits the small group). While the aggregate effort effect is independent of the public-private composition of the contested prize, the strength of the size deterrence effect is increasing in the degree of privateness. Therefore, as long as the choice of sharing rules is unconstrained, the large group is able to fully exploit the

\footnotetext{
${ }^{16}$ Two more possible equilibria characterized by the equilibrium sharing rules presented in Proposition 3 and 5 are the ones such that (i) only the large group decides to restrict the choice of its sharing rule (if $p$ is large enough) and (ii) only the small group decides to restrict the choice of its sharing rule (if $\left.p<p_{1}\right)$.
} 
aggregate effort effect, to the point of excluding the small group from the competition for low levels of privateness of the prize (i.e., monopolization arises). Conversely, for almost purely private contested prizes, the small group, besides being active, even outperforms the large group in terms of winning probabilities (i.e., the GSP arises).

The occurrence of both monopolization and the GSP is driven by the combination of unbounded levels of meritocracy and intermediate degrees of privateness of the contested prize. Insofar as the competition involves groups of individuals, the private or public nature of the prize must be taken into account. Furthermore, considering the unrestricted choice of group sharing rules is clearly relevant to the extent that it would always arise endogenously as an equilibrium outcome regardless of the exact public-private composition of the prize. Yet, as this case is generally pareto-dominated by the case of bounded levels of meritocracy, the two groups would coordinate on the latter if they were ever given the opportunity to collude. The resulting imposed decrease in the strength of the competition, although it does not allow the groups to fully exploit their respective size advantage, would benefit all individuals regardless of their group membership.

We have analyzed the relationship between the size of a group and its performance in terms of its probability of winning the prize. However, another relevant notion of group effectiveness relates its size to per-capita payoffs. As it turns out, whenever meritocracy is unbounded, the members of the small group achieve strictly higher expected utility than the large group's members when the prize is sufficiently private, and from a degree of privateness strictly smaller than the one required for the GSP to arise. In fact, when the prize is sufficiently private so that both groups are active, individual utility is decreasing in the degree of privateness in the large group, while it is increasing in the small group. For both notions of group effectiveness, therefore, the members of the small group always outperform the members of the large group in a competition over a pure private good. 


\section{Appendix}

Proof of Proposition 1. This proof relies on an extension of Ueda (2002). In order to facilitate comparison, let us transform $\alpha_{i}=1-\hat{\alpha}_{i}$.

The expected utility of individual $k=1, \ldots, n_{i}$ in group $i=A, B$ is

$$
\left[\left(1-\hat{\alpha}_{i}\right) \frac{e_{k i}}{\sum_{j=1}^{n_{i}} e_{j i}} p+\hat{\alpha}_{i} \frac{1}{n_{i}} p+(1-p)\right] \frac{E_{i}}{\left(E_{A}+E_{B}\right)} V-e_{k i}
$$

By the Kuhn-Tucker Theorem, the necessary and sufficient conditions to characterize the unique symmetric pure-strategy Nash equilibrium are the following: (i) $e_{k i}=E_{i} / n_{i}$ for each member of group $i=A, B$ and (ii)

$$
\frac{V}{E}\left\{\left(1-\hat{\alpha}_{i}\right) p+\frac{\hat{\alpha}_{i}}{n_{i}} p+(1-p)-\frac{E_{i}}{E}\left[1-p\left(1-\frac{1}{n_{i}}\right)\right]\right\}-1 \leq 0 \text { for all } i
$$

Similarly to Ueda (2002) we can define:

$$
\hat{\gamma}_{i}=\left(1-\hat{\alpha}_{i}\right) p+\frac{\hat{\alpha}_{i}}{n_{i}} p+(1-p)
$$

We also define $\hat{n}_{i}=\frac{1}{1-p\left(1-\frac{1}{n_{i}}\right)}$, which is always positive and larger than one. Then the above necessary and sufficient conditions for the pure strategy Nash equilibrium can be written as

$$
\frac{V}{E}\left(\hat{\gamma}_{i}-\frac{E_{i} \hat{n}_{i}}{E}\right)-1 \leq 0 \quad \text { for } i=A, B
$$

This expression is analogous to expression (2) in Ueda (2002, p. 616), which guarantees that Proposition 1 and Corollary 1 in Ueda (2002) hold in our case. Then, in our two-groups case, from Corollary 1(b), members of group $i$ are inactive if and only if

$$
\hat{\gamma}_{i} \leq \hat{\gamma}_{j}-\frac{1}{\hat{n}_{i}}, \forall i \neq j
$$

Rewriting this condition in terms of $\alpha_{A}, \alpha_{B}$ yields $\chi_{i}\left(\alpha_{A}, \alpha_{B}\right) \geq 0$ in Proposition 1. Solving the system of equations arising from the first-order conditions in the interior and corner solutions, we find the equilibrium effort levels. 
Proof of Proposition 2. By definition the GSP takes place if and only if $E_{A}<E_{B}$. Consider the equilibrium effort levels when both groups are active. Then $\tilde{E}_{A}=n_{A} \hat{e}_{A}<\tilde{E}_{B}=$ $n_{B} \hat{e}_{B}$ can be written as

$$
\frac{p A\left(\alpha_{A}, \alpha_{B}\right) B\left(\alpha_{A}, \alpha_{B}\right)}{C} V>0
$$

where:

$A\left(\alpha_{A}, \alpha_{B}\right)=-\left[n_{B}(1-p)+p\right]\left[n_{A}(1-p)+p+\left(n_{A}-1\right) p \alpha_{A}\right]-\left(n_{B}-1\right)\left[n_{A}(1-p)+p\right] p \alpha_{B}<0$,

$B\left(\alpha_{A}, \alpha_{B}\right)=n_{B}\left(1-2 \alpha_{A}\right)+n_{A}\left[2 n_{B}\left(\alpha_{A}-\alpha_{B}\right)+2 \alpha_{B}-1\right]$

and $C=\left[n_{A}\left(2 n_{B}(p-1)-p\right)-n_{B} p\right]^{2}>0$

The above expression is equal to zero for $p=0$, hence the GSP does not arise in that case. For other values of $p$ we need to consider the sign of each expression. Since $C$ is always positive and $A\left(\alpha_{A}, \alpha_{B}\right)$ is always negative, the GSP arises if and only if $B\left(\alpha_{A}, \alpha_{B}\right)<0$, which can be rearranged as

$$
\alpha_{A}<\frac{n_{A}-n_{B}+2 \alpha_{B} n_{A}\left(n_{B}-1\right)}{2 n_{B}\left(n_{A}-1\right)}
$$

Proof of Proposition 3.

From Proposition 1 and the equilibrium effort levels if both groups are active, the expected utility for group $i=A, B$ is given by

$$
\hat{E U} U_{i}\left(\alpha_{A}, \alpha_{B}\right)=\frac{\left[n_{i} n_{j}+\left(\left(\alpha_{i}-1\right)\left(n_{i}-1\right) n_{j}+\alpha_{j} n_{i}\left(1-n_{j}\right)\right) p\right] V}{n_{i}\left[n_{j} p+n_{i}\left(2 n_{j}(1-p)+p\right)\right]^{2}}\left[n_{i}\left(2 n_{i}-1\right) n_{j}-A p+B\left(n_{i}-1\right) p^{2}\right]
$$

where

$$
\begin{gathered}
A=n_{i}\left(1-\alpha_{j}-n_{i}\right)+n_{j}\left(1-\alpha_{i}\right)+n_{i}\left(4 n_{i}+\alpha_{i}+\alpha_{j}-5\right) n_{j} \\
B=\alpha_{i}\left(n_{j}-1\right)+\alpha_{j}\left(n_{j}-1\right)+\left(n_{i}-1\right)\left(2 n_{j}-1\right)
\end{gathered}
$$

From Proposition 1 and the equilibrium effort levels if only group $i$ is active, the expected utility for group $i$ is given by 


$$
\tilde{E U}{ }_{i}\left(\alpha_{i}\right)=V-\frac{\left(n_{i}-1\right)\left(n_{i}+\alpha_{i}\right)}{n_{i}^{2}} p V
$$

If group $i$ is inactive then from Proposition 1 it must be that $\chi_{i}\left(\alpha_{i}, \alpha_{j}\right) \geqslant 0$. Notice that $\chi_{i}\left(\alpha_{i}, \alpha_{j}\right)+\chi_{j}\left(\alpha_{i}, \alpha_{j}\right)=-n_{A}\left[2 n_{B}(1-p)+p\right]-n_{B} p<0$. Hence if $\chi_{i}\left(\alpha_{i}, \alpha_{j}\right) \geqslant 0$ then $\chi_{j}\left(\alpha_{i}, \alpha_{j}\right)<0$, meaning that if $i$ is inactive $j$ must be active. Therefore if $i$ is inactive it holds that $E U_{i}\left(\alpha_{i}\right)=0$.

Solving $\chi_{j}\left(\alpha_{i 2}\left(\alpha_{j}\right), \alpha_{j}\right)=0$ we define $\alpha_{i 2}\left(\alpha_{j}\right)=\frac{n_{i}}{n_{i}-1}\left[\frac{1-p}{p}+\frac{\left(n_{j}-1\right) \alpha_{j}+1}{n_{j}}\right]$. Given that $\chi_{j}\left(\alpha_{i}, \alpha_{j}\right)$ is strictly increasing with respect to $\alpha_{i}$ it holds that $\chi_{j}\left(\alpha_{i}, \alpha_{j}\right)>0$ for all values $\alpha_{i}>\alpha_{i 2}\left(\alpha_{j}\right)$ and hence $\alpha_{i 2}\left(\alpha_{j}\right)$ is the minimum value of $\alpha_{i}$ that guarantees that members of group $j$ are inactive in equilibrium given any $\alpha_{j}$.

Solving $\chi_{i}\left(\alpha_{i 3}\left(\alpha_{j}\right), \alpha_{j}\right)=0$ we define $\alpha_{i 3}\left(\alpha_{j}\right)=\frac{n_{j} p+n_{i}\left[n_{j}+\alpha_{j} p-\left(\alpha_{j}+1\right) n_{j} p\right]}{\left(1-n_{i}\right) n_{j} p}$. Given that $\chi_{i}\left(\alpha_{i}, \alpha_{j}\right)$ is strictly decreasing with respect to $\alpha_{i}$ then for all $\dot{\alpha}_{i} \in\left[0, \alpha_{i 3}\left(\alpha_{j}\right)\right]$ it is true that $\chi_{i}\left(\dot{\alpha}_{i}, \alpha_{j}\right) \geqslant 0$ and thus group $i$ is inactive.

Comparing the expressions of $\alpha_{i 2}\left(\alpha_{j}\right)$ and $\alpha_{i 3}\left(\alpha_{j}\right)$ we have that $\alpha_{i 2}\left(\alpha_{j}\right)>\alpha_{i 3}\left(\alpha_{j}\right)$ and therefore we can write the expected utility as follows:

$$
E U_{i}\left(\alpha_{i}\right)= \begin{cases}0 & \text { if } 0 \leq \alpha_{i} \leq \alpha_{i 3}\left(\alpha_{j}\right) \\ \hat{E} U_{i}\left(\alpha_{A}, \alpha_{B}\right) & \text { if } \alpha_{i 3}\left(\alpha_{j}\right)<\alpha_{i}<\alpha_{i 2}\left(\alpha_{j}\right) \\ \tilde{E} U_{i}\left(\alpha_{i}\right) & \text { if } \alpha_{i} \geq \alpha_{i 2}\left(\alpha_{j}\right)\end{cases}
$$

Notice that $E U_{i}\left(\alpha_{i}\right)$ is a continuous function since

$$
\lim _{\alpha_{i} \rightarrow \alpha_{i 3}\left(\alpha_{j}\right)} \hat{E U} U_{i}\left(\alpha_{A}, \alpha_{B}\right)=0 \text { and } \lim _{\alpha_{i} \rightarrow \alpha_{i 2}\left(\alpha_{j}\right)} \hat{E U_{i}}\left(\alpha_{A}, \alpha_{B}\right)=\tilde{E U}_{i}\left(\alpha_{i}\right)
$$

Moreover, the second derivative of $\hat{E} U_{i}\left(\alpha_{A}, \alpha_{B}\right)$ is $\frac{2 n_{B}\left(n_{A}-1\right)^{2}\left[n_{B}(p-1)-p\right] p^{2} V}{n_{A}\left[n_{B} p+n_{A}\left(2 n_{B}(1-p)+p\right)\right]^{2}}<0$. Thus, $\hat{E U_{i}}\left(\alpha_{A}, \alpha_{B}\right)$ is a strictly concave function in the unrestricted domain $\alpha_{i} \in(-\infty,+\infty)$ and reaches a global maximum at

$$
\alpha_{i 1}\left(\alpha_{j}\right)=\frac{n_{j}\left[n_{i}(1-p)+p\right]\left[n_{j}\left(2-2 n_{i}(1-p)-3 p\right)-\left(n_{i}-2\right) p\right]-\left(n_{j}-1\right)\left(n_{i}-n_{j}\right) p^{2} \alpha_{j}}{2\left(n_{i}-1\right) n_{j}\left[n_{j}(p-1)-p\right] p}
$$

Finally, notice that $\tilde{E U} U_{i}\left(\alpha_{i}\right)$ is strictly decreasing with respect to $\alpha_{i}$. 
If $\alpha_{i 1}\left(\alpha_{j}\right) \leq \alpha_{i 3}\left(\alpha_{j}\right)$ then for all $\alpha_{i}>\alpha_{i 3}\left(\alpha_{j}\right)$ the expected utility $E U_{i}\left(\alpha_{i}\right)$ is strictly decreasing and hence negative. Therefore, $E U_{i}\left(\alpha_{i}\right)$ takes its maximal value of zero for all values $\dot{\alpha}_{i} \in\left[0, \alpha_{i 3}\left(\alpha_{j}\right)\right]$. Comparing the analytical expressions of $\alpha_{i 1}\left(\alpha_{j}\right)$ and $\alpha_{i 3}\left(\alpha_{j}\right)$ it is true that $\alpha_{i 1}\left(\alpha_{j}\right) \leq \alpha_{i 3}\left(\alpha_{j}\right)$ if and only if

$$
\alpha_{j} \geq \frac{n_{j}}{n_{j}-1} \frac{n_{i}(1-p)+p}{p}=\hat{\alpha}_{j}
$$

If $\alpha_{i 1}\left(\alpha_{j}\right) \geq \alpha_{i 2}\left(\alpha_{j}\right)$ and given that $\tilde{E} U_{i}\left(\alpha_{i}\right)$ is strictly decreasing with respect to $\alpha_{i}$ then $E U_{i}\left(\alpha_{i}\right)$ has a unique global maximum at $\alpha_{i 2}\left(\alpha_{j}\right)$. Comparing the analytical expressions of $\alpha_{i 1}\left(\alpha_{j}\right)$ and $\alpha_{i 2}\left(\alpha_{j}\right)$ it is true that $\alpha_{i 1}\left(\alpha_{j}\right) \geq \alpha_{i 2}\left(\alpha_{j}\right)$ if and only if

$$
\alpha_{j} \leq \frac{n_{j}\left[n_{i}(1-p)+3 p-2\right]-2 p}{\left(n_{j}-1\right) p}=\tilde{\alpha}_{j}
$$

If $\alpha_{i 3}\left(\alpha_{j}\right)<\alpha_{i 1}\left(\alpha_{j}\right)<\alpha_{i 2}\left(\alpha_{j}\right)$ then $E U_{i}\left(\alpha_{i}\right)$ has a unique global maximum at $\alpha_{i 1}\left(\alpha_{j}\right)$.

Summing up, the best response of group $i$ can be written as:

$$
\alpha_{i}\left(\alpha_{j}\right)= \begin{cases}\alpha_{i 2}\left(\alpha_{j}\right) & \text { for } \alpha_{j} \leq \tilde{\alpha}_{j} \\ \alpha_{i 1}\left(\alpha_{j}\right) & \text { for } \tilde{\alpha}_{j}<\alpha_{j}<\hat{\alpha}_{j} \\ \dot{\alpha}_{i} \in\left[0, \alpha_{i 3}\left(\alpha_{j}\right)\right] & \text { for } \alpha_{j} \geq \hat{\alpha}_{j}\end{cases}
$$

We now consider the nine possible combinations of the two groups' best responses:

Case 1: $i=A, B$ plays $\alpha_{i 1}\left(\alpha_{j}\right)$

If $i=A, B$ plays $\alpha_{i 1}\left(\alpha_{j}\right)$, it yields the following sharing rule:

$$
\alpha_{i}=\frac{n_{i}}{\left(n_{i}-1\right)} \frac{2 n_{i} n_{j}\left(n_{i}-1\right)+A_{i} p+B_{i} p^{2}}{p\left[2 n_{i} n_{j}-p\left(n_{i}\left(2 n_{j}-1\right)-n_{j}\right)\right]}
$$

where $A_{i}=n_{i} n_{j}\left(9-4 n_{i}\right)-n_{j}\left(n_{j}+2\right)-2 n_{i}$ and $B_{i}=n_{i} n_{j}\left(2 n_{i}-7\right)+n_{j}\left(n_{j}+3\right)+3 n_{i}-2$.

From the best responses, this is an equilibrium if and only if $\tilde{\alpha}_{i}<\alpha_{i}<\hat{\alpha}_{i}$ for $i=A, B$, which is true as long as 


$$
p>\frac{n_{B}\left(n_{A}-n_{B}-1\right)}{1+n_{B}\left(n_{A}-n_{B}-1\right)}=p_{1}
$$

Hence, both groups being active is an equilibrium if and only if $p>p_{1}$.

Case 2: $i=A, B$ plays $\alpha_{i 2}\left(\alpha_{j}\right)$

If $i=A, B$ plays $\alpha_{i 2}\left(\alpha_{j}\right)$, there is no solution, as the best responses are two parallel lines with positive slope.

Case 3: $A$ plays $\alpha_{A 1}\left(\alpha_{B}\right)$ and $B$ plays $\alpha_{B 2}\left(\alpha_{A}\right)$

If $A$ plays $\alpha_{A 1}\left(\alpha_{B}\right)$ and $B$ plays $\alpha_{B 2}\left(\alpha_{A}\right)$, it yields the following sharing rules:

$$
\begin{gathered}
\alpha_{A}=1+n_{A}\left(\frac{1}{p}-1\right) \\
\alpha_{B}=\frac{n_{B}\left[n_{A}(1-p)+p\right]}{\left(n_{B}-1\right) p}
\end{gathered}
$$

From the best responses, this is an equilibrium if and only if $\alpha_{A} \leqslant \tilde{\alpha}_{A}$ and $\tilde{\alpha}_{B}<$ $\alpha_{B}<\hat{\alpha}_{B}$. As the sharing rules above are such that $\alpha_{B}>\hat{\alpha}_{B}$, the latter condition is never satisfied. Hence we do not reach an equilibrium.

Case 4: $A$ plays $\alpha_{A 2}\left(\alpha_{B}\right)$ and $B$ plays $\alpha_{B 1}\left(\alpha_{A}\right)$

If $A$ plays $\alpha_{A 2}\left(\alpha_{B}\right)$ and $B$ plays $\alpha_{B 1}\left(\alpha_{A}\right)$, it yields the following sharing rules:

$$
\begin{gathered}
\alpha_{A}=\frac{n_{A}\left[n_{B}(1-p)+p\right]}{\left(n_{A}-1\right) p} \\
\alpha_{B}=1+n_{B}\left(\frac{1}{p}-1\right)
\end{gathered}
$$

From the best responses, this is an equilibrium if and only if $\alpha_{B} \leqslant \tilde{\alpha}_{B}$ and $\tilde{\alpha}_{A}<$ $\alpha_{A}<\hat{\alpha}_{A}$. As the sharing rules above are such that $\alpha_{A}>\hat{\alpha}_{A}$, the latter condition is never satisfied. Hence we do not reach an equilibrium.

Case 5: $A$ plays $\alpha_{A 1}\left(\alpha_{B}\right)$ and $B$ plays $\dot{\alpha}_{B} \in\left[0, \alpha_{B 3}\left(\alpha_{A}\right)\right]$

If $A$ plays $\alpha_{A 1}\left(\alpha_{B}\right)$ and $B$ plays $\dot{\alpha}_{B} \in\left[0, \alpha_{B 3}\left(\alpha_{A}\right)\right]$, we are at an equilibrium if and only if $\alpha_{A} \geqslant \hat{\alpha}_{A}$ and $\tilde{\alpha}_{B}<\alpha_{B}<\hat{\alpha}_{B}$, which is never satisfied. Let $\alpha_{A}=\alpha_{A 1}\left(\alpha_{B}\right)$. Then, the condition $\alpha_{B}<\alpha_{B 3}\left(\alpha_{A}\right)$ reduces to $\alpha_{B}<\tilde{\alpha}_{B}$, hence we reach a contradiction. 
Case 6: $A$ plays $\dot{\alpha}_{A} \in\left[0, \alpha_{A 3}\left(\alpha_{B}\right)\right]$ and $B$ plays $\alpha_{B 1}\left(\alpha_{A}\right)$

If $A$ plays $\dot{\alpha}_{A} \in\left[0, \alpha_{A 3}\left(\alpha_{B}\right)\right]$ and $B$ plays $\alpha_{B 1}\left(\alpha_{A}\right)$, we are at an equilibrium if and only if $\tilde{\alpha}_{A}<\alpha_{A}<\hat{\alpha}_{A}$ and $\alpha_{B} \geqslant \hat{\alpha}_{B}$, which is never satisfied. Let $\alpha_{B}=\alpha_{B 1}\left(\alpha_{A}\right)$. Then, the condition $\alpha_{A}<\alpha_{A 3}\left(\alpha_{B}\right)$ reduces to $\alpha_{A}<\tilde{\alpha}_{A}$, hence we reach a contradiction.

Case 7: $i=A, B$ plays $\dot{\alpha}_{i} \in\left[0, \alpha_{i 3}\left(\alpha_{j}\right)\right]$

If $i=A, B$ plays $\dot{\alpha}_{i} \in\left[0, \alpha_{i 3}\left(\alpha_{j}\right)\right]$, we immediately reach a contradiction, as $\alpha_{A} \leqslant$ $\alpha_{A 3}\left(\alpha_{B}\right)$ and $\alpha_{B} \leqslant \alpha_{B 3}\left(\alpha_{A}\right)$ cannot hold simultaneously. The condition $\alpha_{A} \leqslant \alpha_{A 3}\left(\alpha_{B}\right)$ is equivalent to

$$
\alpha_{B} \geqslant \frac{n_{B}\left[n_{A}-\left(1-\alpha_{A}\right)\left(n_{A}-1\right) p\right]}{n_{A}\left(n_{B}-1\right) p}
$$

Then, we have that

$$
\alpha_{B 3}\left(\alpha_{A}\right) \geqslant \frac{n_{B}\left[n_{A}-\left(1-\alpha_{A}\right)\left(n_{A}-1\right) p\right]}{n_{A}\left(n_{B}-1\right) p} \text { if and only if } p \geqslant \frac{2 n_{A} n_{B}}{2 n_{A} n_{B}-n_{A}-n_{B}}>1
$$

Hence we reach a contradiction.

Case 8: $A$ plays $\alpha_{A 2}\left(\alpha_{B}\right)$ and $B$ plays $\dot{\alpha}_{B} \in\left[0, \alpha_{B 3}\left(\alpha_{A}\right)\right]$

If $A$ plays $\alpha_{A 2}\left(\alpha_{B}\right)$ and $B$ plays $\dot{\alpha}_{B} \in\left[0, \alpha_{B 3}\left(\alpha_{A}\right)\right]$, we are at an equilibrium if and only if $\alpha_{A} \geqslant \hat{\alpha}_{A}$ and $\alpha_{B} \leqslant \tilde{\alpha}_{B}$, which holds if and only if $\alpha_{B} \in\left[\frac{n_{B}(1-p)+p}{p}, \tilde{\alpha}_{B}\right]$ and $p \leqslant p_{1}$.

Case 9: $A$ plays $\dot{\alpha}_{A} \in\left[0, \alpha_{A 3}\left(\alpha_{B}\right)\right]$ and $B$ plays $\alpha_{B 2}\left(\alpha_{A}\right)$

If $A$ plays $\dot{\alpha}_{A} \in\left[0, \alpha_{A 3}\left(\alpha_{B}\right)\right]$ and $B$ plays $\alpha_{B 2}\left(\alpha_{A}\right)$, we are at an equilibrium if and only if $\alpha_{A} \leqslant \tilde{\alpha}_{A}$ and $\alpha_{B} \geqslant \hat{\alpha}_{B}$, which is never satisfied. Let $\alpha_{B}=\alpha_{B 2}\left(\alpha_{A}\right)$. Then, $\alpha_{B} \geqslant \hat{\alpha}_{B}$ reduces to $\alpha_{A} \geqslant\left[n_{A}(1-p)+p\right] / p$. As $\tilde{\alpha}_{A}<\left[n_{A}(1-p)+p\right] / p$, the conditions for an equilibrium are never satisfied.

Proof of Proposition 4. Clearly, if the small group is inactive, the GSP cannot occur. If $p>p_{1}$, from Proposition 2, the GSP arises if and only if 


$$
\alpha_{A}<\frac{n_{A}-n_{B}+2 \alpha_{B} n_{A}\left(n_{B}-1\right)}{2 n_{B}\left(n_{A}-1\right)}
$$

Substituting for the equilibrium value of $\alpha_{i}(i=A, B)$ from Proposition 3, the above condition reduces to

$$
\frac{\left(n_{A}-n_{B}\right)\left[2 n_{A} n_{B}(1-p)-p\right]}{2\left(n_{A}-1\right) n_{B} p}<0
$$

which holds if and only if $p>\frac{2 n_{A} n_{B}}{1+2 n_{A} n_{B}}=p_{G S P}$.

Proof of Proposition 5. We can obtain group $i$ 's best response with restricted sharing rules by adding the constraint $\alpha_{i} \leq 1$ for $i=A, B$ in the best response with unrestricted sharing rules derived in the proof of Proposition 3.

First, observe that $\hat{\alpha}_{i}>1$ for $i=A, B$ and $\forall p \in[0,1]$. Therefore, $\dot{\alpha}_{i}$ is not part of the best response with restricted sharing rules. As shown in the proof of Proposition 3, $\alpha_{i 1}\left(\alpha_{j}\right)$ is chosen over $\alpha_{i 2}\left(\alpha_{j}\right)$ if and only if $\alpha_{i 1}\left(\alpha_{j}\right)<\alpha_{i 2}\left(\alpha_{j}\right)$. By taking this into account together with the new restriction $\alpha_{i} \leq 1$, one can write the best response for $i=A, B$ and $j \neq i$ as:

$$
\alpha_{i}\left(\alpha_{j}\right)=\min \left\{\alpha_{i 1}\left(\alpha_{j}\right), \alpha_{i 2}\left(\alpha_{j}\right), 1\right\}
$$

To find the solution we have to consider the nine combinations that arise from the previous best response. We can easily eliminate several combinations:

- If $i=A, B$ plays $\alpha_{i 1}\left(\alpha_{j}\right)$, the sharing rule of each group is the one obtained in Case 1 in the proof of Proposition 3. As the value of $\alpha_{B}$ always exceeds one we can discard this combination as a solution.

- If $i=A, B$ plays $\alpha_{i 2}\left(\alpha_{j}\right)$, there is no solution, as the best responses are parallel lines (Case 2 in the proof of Proposition 3).

- From cases 3 and 4 in the proof of Proposition 3, if $i$ plays $\alpha_{i 1}\left(\alpha_{j}\right)$ and $j$ plays 
$\alpha_{j 2}\left(\alpha_{i}\right)$, we obtain that

$$
\alpha_{i}=\frac{n_{i}\left[n_{j}(1-p)+p\right]}{\left(n_{i}-1\right) p}>1
$$

Thus, $i$ and $j$ playing $\alpha_{i 1}\left(\alpha_{j}\right)$ and $\alpha_{j 2}\left(\alpha_{i}\right)$ for $i=A, B$ and $j \neq i$ never constitutes an equilibrium.

- As $\alpha_{i 2}(1)=\frac{n_{B}}{\left(n_{B}-1\right) p}>1$ for $i=A, B$, there is no solution with $i$ and $j$ playing $\alpha_{i 2}\left(\alpha_{j}\right)$ and $\alpha_{j}=1$ for $i=A, B$ and $j \neq i$.

- Let $\alpha_{A}=1$ and $\alpha_{B}\left(\alpha_{A}\right)=\alpha_{B 1}(1)$. By evaluating $\alpha_{B 1}(1)$ at $p=1$ we obtain that $\alpha_{B}(1)=\frac{2 n_{A} n_{B}-n_{A}-n_{B}}{2 n_{A}\left(1-n_{B}\right)}>1$. Then, as $\frac{\partial \alpha_{B 1}(1)}{\partial p}=-\frac{2 n_{B}\left(n_{A}+p-n_{A} p\right)^{2}+\left(n_{A}-n_{B}\right) p^{2}}{2 p^{2}\left(n_{A}+p-n_{A} p\right)^{2}}<0$ for all $p \in(0,1]$, it follows that $\alpha_{B 1}(1)>1$ for any $p \in[0,1]$.

After eliminating the previous seven combinations we are now left with the two cases where $\alpha_{B}=1$ and $\alpha_{A}=\min \left\{\alpha_{A 1}(1), 1\right\}$. By substitution of $\alpha_{B}=1$ we obtain the analytical value of $\alpha_{A 1}(1)$ :

$$
\alpha_{A 1}(1)=\frac{n_{A}^{2} n_{B}\left[2 n_{B}(1-p)+p\right](1-p)-n_{B} p\left[2 n_{B}(1-p)+p\right]-n_{A}\left[2 n_{B}(1-2 p) p+p^{2}+n_{B}^{2}(2+p(5 p-7))\right]}{2\left(n_{A}-1\right) n_{B}\left[n_{B}(1-p)+p\right] p}
$$

Solving $\alpha_{A 1}(1)=1$ with respect to $p$ yields

$$
p_{1,2}=\frac{1}{2}\left\{\frac{n_{A} n_{B}\left[2-5 n_{B}+n_{A}\left(4 n_{B}-1\right)\right]}{n_{B}+n_{A}\left[n_{B}\left[2-3 n_{B}+n_{A}\left(2 n_{B}-1\right)\right]-1\right]} \pm \sqrt{\frac{n_{A} n_{B}^{2}\left[8 n_{B}+n_{A}\left[n_{A}^{2}+\left(n_{B}-12\right) n_{B}+2 n_{A}\left(2+n_{B}\right)-4\right]\right]}{\left[n_{B}+n_{A}\left[n_{B}\left(2-3 n_{B}+n_{A}\left(2 n_{B}-1\right)\right)-1\right]\right]^{2}}}\right\}
$$

As $p_{1}$ (with the plus sign) is larger than one, we further ignore it. Thus we let $\stackrel{\circ}{p}=p_{2}<$ 1 be the relevant value such that $\alpha_{A 1}(1)=1$. As $\frac{\partial \alpha_{A 1}(1)}{\partial p}=\frac{p^{2}\left(n_{A}-n_{B}\right)-2 n_{A}\left(n_{B}+p-n_{B} p\right)^{2}}{p^{2} 2\left(n_{B}+p-n_{B} p\right)^{2}}<0$ for all $p \in(0,1]$, it follows that $\alpha_{A 1}(1)>1$ for any $p \in[0, \stackrel{\circ}{p})$. Therefore, at the unique equilibrium we have

- $\alpha_{A}=\alpha_{B}=1$ for $p \in(0, \stackrel{p}{p}]$

- $\alpha_{A}=\alpha_{A 1}(1)$ and $\alpha_{B}=1$ for $p \in(\stackrel{\circ}{p}, 1]$ 
Substituting these equilibrium values in the relevant expression obtained in Proposition 1 , we find that $\chi_{i}\left(\alpha_{A}, \alpha_{B}\right)<0$ for $i=A, B$ and for all $p \in(0,1]$. Hence in equilibrium both groups are active.

Proof of Proposition 6. From Proposition 2, for any $p \in(0,1]$ the GSP arises if and only if $\alpha_{A}<\frac{n_{A}-n_{B}+2 \alpha_{B} n_{A}\left(n_{B}-1\right)}{2 n_{B}\left(n_{A}-1\right)}$. We know from Proposition 5 that $\alpha_{A}=\alpha_{B}=1$ for $p \in(0, \stackrel{p}{p}]$, hence the GSP arises if and only if $\frac{n_{A}-n_{B}}{n_{A} n_{B}-n_{B}}<0$ which never holds given that $n_{A}>n_{B}$.

For $p \in(\stackrel{\circ}{p}, 1]$ we know that $\alpha_{B}=1$ and $\alpha_{A}=\alpha_{A 1}(1)$. Taking the equilibrium value of $\alpha_{A}(1)$ for $p=1$ yields $\alpha_{A}(1)=\frac{2 n_{A} n_{B}-n_{A}-n_{B}}{2 n_{B}\left(n_{A}-1\right)}$. From Proposition 2, the GSP arises at $p=1$ if and only if $\frac{2 n_{A} n_{B}-n_{A}-n_{B}}{2 n_{B}\left(n_{A}-1\right)}<\frac{n_{A}-n_{B}+2 n_{A}\left(n_{B}-1\right)}{2 n_{B}\left(n_{A}-1\right)}$ which never holds given that $n_{A}>n_{B}$. Thus, the GSP does not arise at $p=1$. As we have shown that $\frac{\partial \alpha_{A 1}(1)}{\partial p}<0$ for all $p \in(0,1]$, it follows that the GSP does not arise for any $p \in(\stackrel{\circ}{p}, 1)$. 


\section{References}

BAIK, K. H. (1993): "Effort levels in contests: The public-good prize case," Economics Letters, 41, 363-367.

(1994): "Winner-help-loser group formation in rent-seeking contests," Economics \& Politics, 6, 147-162.

(2008): "Contests with group-specific public-good prizes," Social Choice and Welfare, 30, 103-117.

(2014): "Endogenous Group Formation in Contests: Unobservable Sharing Rules," Working Paper.

BAIK, K. H. AND D. LEE (2012): "Do rent-seeking groups announce their sharing rules?" Economic Inquiry, 50, 348-363.

BAIK, K. H. AND S. LEE (1997): "Collective rent seeking with endogenous group sizes," European Journal of Political Economy, 13, 121-130.

(2001): "Strategic groups and rent dissipation," Economic Inquiry, 39, 672-684.

(2007): "Collective rent seeking when sharing rules are private information," European Journal of Political Economy, 23, 768-776.

BAIK, K. H. AND J. F. Shogren (1995): "Competitive-share group formation in rentseeking contests," Public choice, 83, 113-126.

Corchón, L. C. (2007): "The theory of contests: a survey," Review of Economic Design, $11,69-100$.

Davis, D. D. And R. J. Reilly (1999): "Rent-seeking with non-identical sharing rules: An equilibrium rescued," Public Choice, 100, 31-38.

Esteban, J.-M. And D. RAY (2001): "Collective action and the group size paradox," American Political Science Review, 95, 663-672.

Flamand, S. And O. Troumpounis (2014): "Prize-sharing rules in collective rent seeking," Working Paper.

Gürtler, O. (2005): "Rent seeking in sequential group contests," Bonn Economic Discussion Papers \#4\%.

Katz, E., S. Nitzan, And J. Rosenberg (1990): "Rent-seeking for pure public goods," Public Choice, 65, 49-60.

LEE, S. (1995): "Endogenous sharing rules in collective-group rent-seeking," Public Choice, 85, 31-44.

Lee, S. And J. Hyeong Kang (1998): "Collective contests with externalities," European Journal of Political Economy, 14, 727-738. 
Nitzan, S. (1991): "Collective rent dissipation," Economic Journal, 101, 1522-1534.

Nitzan, S. AND K. UEDA (2011): "Prize sharing in collective contests," European Economic Review, 55, 678-687.

— (2013): "Intra-group heterogeneity in collective contests," Social Choice and Welfare, forthcoming.

- (2014): "Cost Sharing in Collective Contests," CESifo Working Paper Series.

NoH, S. J. (1999): "A general equilibrium model of two group conflict with endogenous intra-group sharing rules," Public Choice, 98, 251-267.

Olson, M. (1965): The Logic of Collective Action, Harvard MA: Harvard University Press.

Pecorino, P. And A. Temimi (2008): "The group size paradox revisited," Journal of Public Economic Theory, 10, 785-799.

Riaz, K., J. F. Shogren, And S. R. Johnson (1995): "A general model of rent seeking for public goods," Public Choice, 82, 243-259.

UEDA, K. (2002): "Oligopolization in collective rent-seeking," Social Choice and Welfare, $19,613-626$.

Ursprung, H. W. (1990): "Public goods, rent dissipation, and candidate competition," Economics \& Politics, 2, 115-132.

VAzQuez, A. (2014): "Sharing the effort costs in collective contests," Available at SSRN. 